\title{
Spinal Eumycetoma: A Rare Cause of Lumbar Canal Stenosis and Significance of "Dot in Circle"
}

\author{
Radha Sarawagi $\quad$ jitendra Sharma ${ }^{1} \quad$ Rajesh Malik $^{1} \quad$ Aman Kumar ${ }^{1}$ \\ 1Department of Radio Diagnosis and Imaging, All India Institute of \\ Medical Sciences, Bhopal, Madhya Pradesh, India

\begin{abstract}
Address for correspondence Dr. Radha Sarawagi, Department of Radiodiagnosis and Imaging, All India Institute of Medical Sciences, Bhopal 462 020, Madhya Pradesh, India (e-mail: radhasanjeevgupta@gmail.com).
\end{abstract}

Indian J Radiol Imaging 2021;31:468-471.

\begin{abstract}
Mycetoma is a chronic granulomatous disease that is more common in tropical regions with predominant involvement of foot. Spinal mycetoma presenting as lumbar canal stenosis is extremely rare. We hereby present a case of fungal eumycetoma of verte-

Keywords

- dot-in-circle

- eumycetoma

- lumbar spine

- magnetic resonance imaging

- ultrasonography bral column in a 42-year-old male who presented with chronic progressive low back pain and features of lumbar canal stenosis without any skin swelling or discharging sinuses. The "dot-in-circle" sign, a highly specific magnetic resonance imaging (MRI) and ultrasonography (USG) sign of mycetoma has been described in the literature as a pathognomonic feature of mycetoma involving the musculoskeletal system. We describe the importance of characteristic imaging features with dot in circle sign in the diagnosis of eumycetoma of lumbar spine.
\end{abstract}

\section{Introduction}

Mycetoma is a chronic inflammatory disease affecting predominantly the skin and subcutaneous tissue with deeper extension to involve muscles and bones. It is caused by either bacteria or true fungi (eumycetoma). It commonly occurs in tropical and subtropical countries. WHO has recently recognized this condition as neglected tropical disease. ${ }^{1,2}$ The disease commonly involves dorsum of the foot; however, involvement of other organs in the body such as the lower legs, hands, head, neck, chest, shoulders, and arms is also seen. Cranial and spinal involvement only has rarely been reported. ${ }^{3-5}$ The clinical triad of pain, swelling, and discharging sinuses containing granules may not be present in early cases and deep-seated disease. Failure to diagnose the case early leads to significant deformity and morbidity. Recognition of typical imaging features results in early diagnosis and facilitate appropriate management. ${ }^{6}$

published online July 27,2021
DOI https://doi.org/

10.1055/s-0041-1734331 ISSN 0971-3026

\section{Case History}

A 42-year-old male, a farmer, presented to the neurosurgery OPD with complaints of bilateral gradual onset paraparesis and paresthesia, with difficulty in micturition for the past 3 years. There was past history of right lower limb swelling 2 years back, which was managed conservatively. There was also a history of perianal region fistula or sinus, for which incision and drainage were done by a local practitioner. One and a half year back, the patient had computed tomography (CT) scan and magnetic resonance imaging (MRI) of lumbar spine elsewhere which was reported as multilevel infective spondylodiskitis. CT-guided biopsy from right paravertebral soft tissue was performed, which showed fibromuscular tissue and inflammatory cells. No organism could be identified. Patient was started on empirical antitubercular treatment, to which patient reported no improvements.

CT showed diffuse sclerotic marrow changes in lower dorsal and lumbosacral spine and pelvic bones with multiple small

(C) 2021. Indian Radiological Association.

This is an open access article published by Thieme under the terms of the Creative Commons Attribution-NonDerivative-NonCommercial-License, permitting copying and reproduction so long as the original work is given appropriate credit. Contents may not be used for commercial purposes, or adapted, remixed, transformed or built upon. (https://creativecommons.org/licenses/by-nc-nd/4.0/).

Thieme Medical and Scientific Publishers Private Ltd. A-12, Second Floor, Sector -2, NOIDA -201301, India 
cavitating lesions. There was a loss of L1-L2 intervertebral disk space with bony ankylosis (-Fig. 1).

MRI in our institute showed sclerotic and edematous marrow changes, involving the lumbar spine and sacrum with multiple small round lytic lesions. There was also evidence of conglomerated round soft tissue lesions involving paravertebral muscles, subcutaneous tissue of back, and extending within the spinal canal into epidural space and causing compression of thecal sac. The lesions were T2 hyperintense with a peripheral low-signal intensity rim and hypointense septae. Some of these hyperintense lesions showed central tiny hypointensities, giving the appearance of "dot in circle" (-Fig. 2A and B ).On T1-weighted images the lesions appeared isointense with hypointense rim and showed heterogeneous post-contrast enhancement. There was also evidence of intradural extension with arachnoiditis (-Fig. 3A and $\mathbf{B}$ ). There were multiple tortuous vascular channels in right perinephric region due to external and common iliac vein thrombosis.

High-resolution ultrasonography (USG) of lower back region showed multiple discrete and conglomerated, nodular hypoechoic lesions in subcutaneous tissues and paraspinal muscles showing central echogenic mobile granule like contents giving "dot-in-circle" appearance (-Fig. 4). Based on imaging findings possibility of eumycetoma was considered. USG-guided true cut biopsy was done from the subcutaneous lesions in lower back, targeting on central echogenic part. Multiple gray-white soft tissue pieces were obtained with multiple black granules.

Microbiology specimen under $\mathrm{KOH}$ mount showed fungal hyphae, abundant hyaline hyphae, and few melanized hyphae. Histopathological examination with Hematoxylin and Eosin (H\&E) staining and Periodic-acid-Schiff (PAS) staining showed fibromuscular tissue along with brownblack-pigmented colonies of fungal hyphae showing septa and branching and embedded in a matrix like material along with the foci of necrosis and inflammatory cells suggesting eumycetoma (- Figs. 5A and B ).

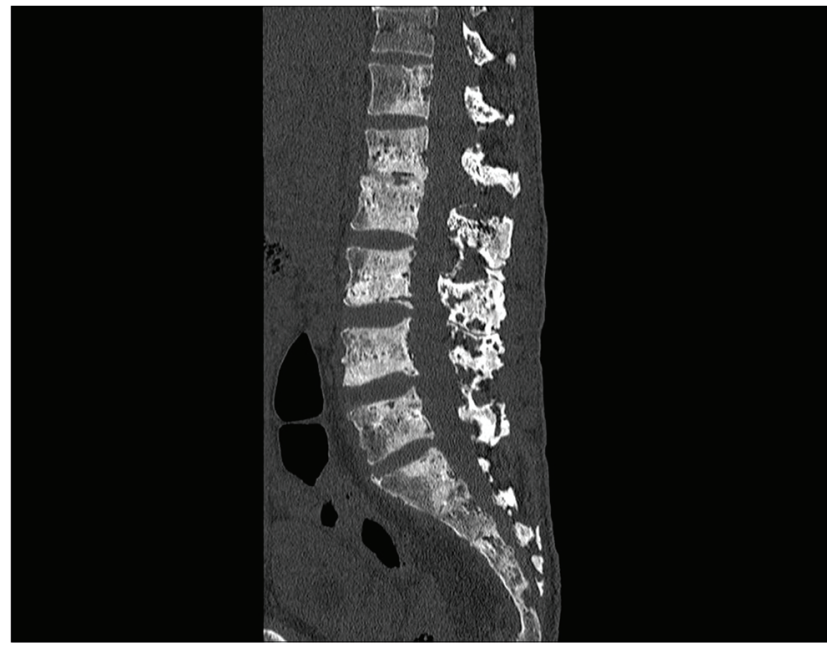

Fig. 1 Non-contrast sagittal CT scan of lumbar spine shows diffuse sclerotic changes in vertebral bodies and posterior elements with multiple lytic lesions. CT, computed tomography.

\section{Discussion}

Diagnosis of mycetoma is usually suspected when patients present with painless subcutaneous nodules, multiple cutaneous sinuses from which a purulent granular exudate may be discharged. The grains are visualized with H\&E staining or by special staining like PAS and Grocott-Gomori silver staining. On histology, the lesion consists of "grains" of fungal hyphae or bacteria in microabscesses within a granulomatous fibrous-tissue reaction. ${ }^{7}$ Early laboratory diagnosis, before the appearance of the sinuses and grains, is difficult. Deeper involvement and absence of discharging sinuses
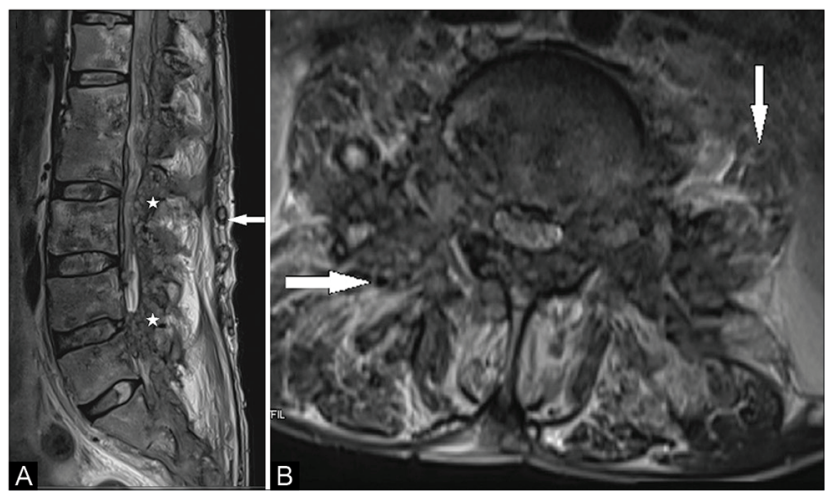

Fig. 2 MRI of lumbar spine (A) T2W sagittal image shows diffuse marrow changes and multiple small round lytic lesions. Diffuse, conglomerated, and discrete round T2 hyperintense lesions with peripheral low-signal-intensity rim, seen in subcutaneous plane (arrow) and in epidural soft tissue (stars) compressing the thecal sac. (B) T2W axial images at vertebral level show hyperintense lesions in paravertebral soft tissue, showing tiny hypointensities within, giving the appearance of "dot-in-circle" (arrows). MRI, magnetic resonance imaging.

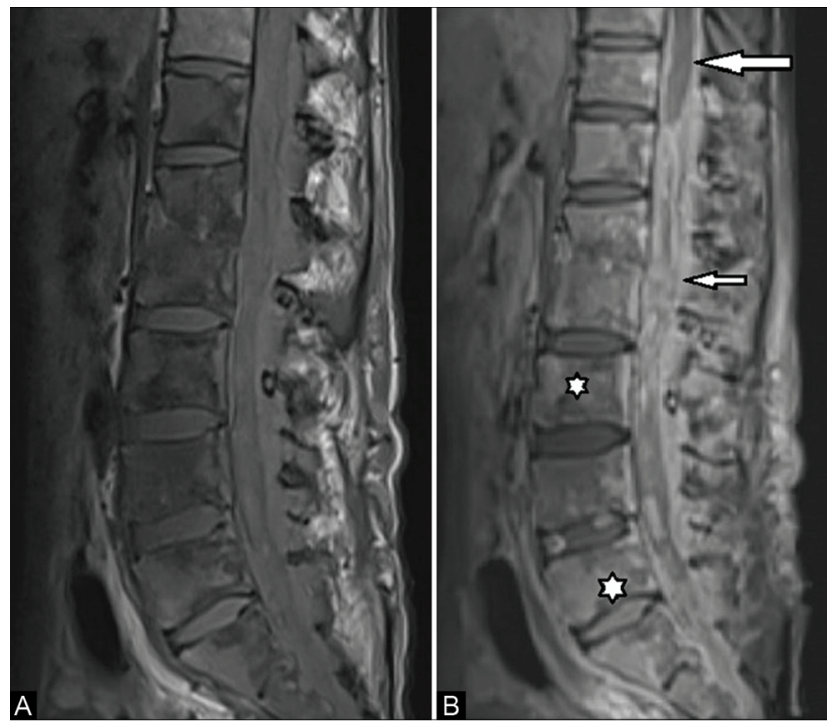

Fig. 3 T1-weighted non-contrast sagittal MRI images (A) show diffuse marrow changes in vertebral bodies and posterior elements of involved lumbosacral spine. (B) Post contrast T1-weighted fat suppressed sagittal images show enhancing marrow edema (stars) and enhancing soft tissues in prevertebral and epidural space (small arrow) along meninges (large arrow). MRI, magnetic resonance imaging. 


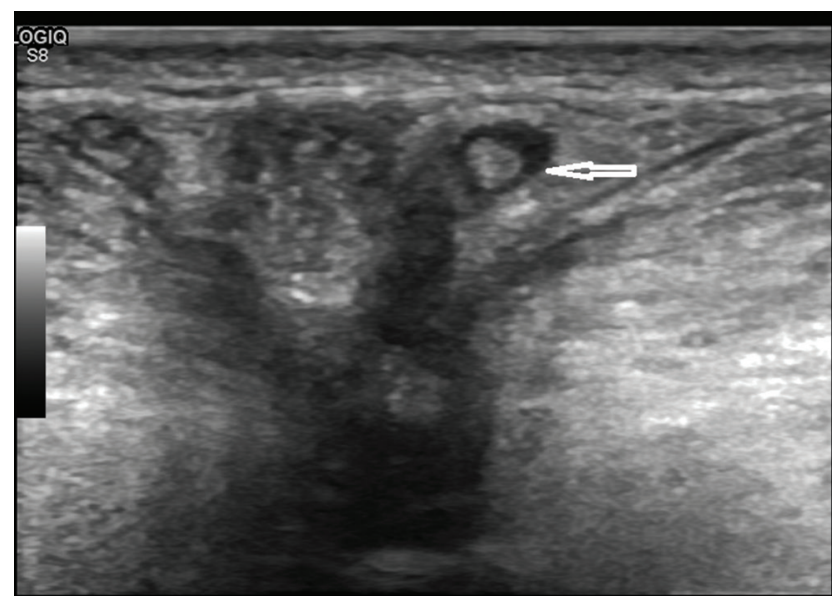

Fig. 4 Ultrasound image of the back with eumycetoma shows multiple hypoechoic lesions containing small, sharp, and hyperechoic foci (arrow).

often leads to misdiagnosis as tumor, tuberculosis, and other chronic bacterial infection as happened in our case. ${ }^{8}$

MRI and USG are helpful in the early diagnosis and in deep-seated lesions without discharging sinuses and help in discriminating mycetoma from other conditions. Presence of conglomerated lesions with "dot-in-circle" sign is very well seen in most of the cases of mycetoma of foot and highly specific to diagnose mycetoma on both ultrasonography and MRI. The high signal area on T2W or short tau inversion recovery MRI represents granulomatous tissue or microabscesses and hypointense septa and wall represent fibrous tissue. The tiny hypointense dots are represented by mycetoma grains. ${ }^{9}$ Similarly on sonography the grain appears as hyperechoic foci within hypoechoic granulation tissue. ${ }^{10}$ The grain of eumycetoma is more sharply hyper-reflective as compared with grain of actinomycetoma. ${ }^{11}$

Involvement of bone is noted due to contiguous spread of soft tissue involvement. Plain radiography or computed tomographic findings in skeletal mycetoma commonly reveal sclerosis, cortical erosions, cavities, periosteal reactions, and bone expansions. ${ }^{12}$ The lesions of actinomycetoma are numerous and smaller in size giving moth-eaten pattern. ${ }^{13,14}$

Spinal involvement of eumycetoma is extremely rare. ${ }^{8}$ Ahmed et al described a case of gluteal eumycetoma with deeper extension to pelvis and lumbosacral spine even after antifungal treatment and surgery. ${ }^{4}$ In another case of spinal cord compression and paraplegia, spinal mycetoma was found on surgery and histopathology. No skin or subcutaneous involvement was found and hematogenous spread was suspected. On histopathology there was evidence of vascular invasion. ${ }^{3,8}$ In our patient there was an involvement of pelvic bones and soft tissue with right external iliac vein thrombosis. There was also a history of surgery at perianal region due to some fistula or discharging sinus which might be the primary site of involvement. Raval and colleagues reported a case of perineal involvement by mycetoma with multiple discharging sinuses. ${ }^{15}$

Treatment of eumycetoma is challenging. Combined medical and surgical approach is required for effective outcome.

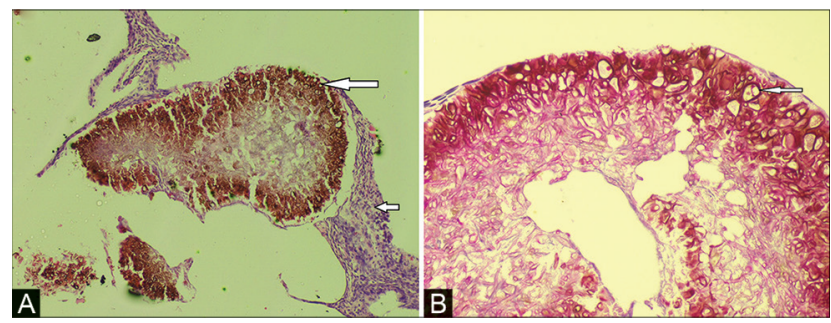

Fig. 5 Histopathological examination with (A) Hematoxylin \& Eosin (H\&E) stain in low power microscope shows brown-black pigmented fungal colonies (large arrow) showing septa and branching, embedded in a matrix-like material (star) with the foci of necrosis and inflammatory cells. (B) Periodic-acid-Schiff (PAS) staining in high power microscope shows multiple club-shaped fungal hyphae. This is an open access journal, and the articles are distributed under the terms of the Creative Commons Attribution-NonCommercial-ShareAlike 4.0 License, which allows others to remix, tweak, and build upon the work noncommercially, as long as appropriate credit is given, and the new creations are licensed under the identical terms.

Triazole group of antifungal medications like itraconazole, voriconazole are the treatment of choice and require prolonged medication for 1 to 2 years. ${ }^{16}$

\section{Conclusion}

Knowledge about this rare but clinically important pathology is important to reach the definitive diagnosis as lack of familiarity with the characteristic imaging features results in delay in the diagnosis and treatment and significant morbidity. Isolation of causative organisms from inflammatory granulation tissue can be difficult particularly in deep-seated lesion without discharging sinuses. During USG-guided biopsy, special care should be taken to include the central echogenic component to get better results.

\section{Declaration of Patient Consent}

The authors certify that they have obtained all appropriate patient consent forms. In the form the patient(s) has/have given his/her/their consent for his/her/their images and other clinical information to be reported in the journal. The patients understand that their names and initials will not be published, and due efforts will be made to conceal their identity, but anonymity cannot be guaranteed.

\section{Financial Support and Sponsorship \\ None.}

\section{Conflict of Interest}

None declared.

\section{References}

1 Verma P, Jha A. Mycetoma: reviewing a neglected disease. Clin Exp Dermatol 2019;44(2):123-129

2 Zijlstra EE, van de Sande WW, Welsh O, Mahgoub ES, Goodfellow M, Fahal AH. Mycetoma: a unique neglected tropical disease. Lancet Infect Dis 2016;16(1):100-112

3 Arbab MA, el Hag IA, Abdul Gadir AF, el-R SH. Intraspinal mycetoma: report of two cases. Am J Trop Med Hyg 1997;56(1):27-29 
4 Ahmed A, Suleiman SH, Fahal AH. Aggressive uncontrolled gluteal eumycetoma invading the pelvic cavity and the spinal cord. Khartoum Med J 2014;7:1005-1007

5 Mohamed Ahmed R, Ezaldeen EA, Wadella ES, El Dawi N, Hassan Fahal A. Cervical spinal cord compression: a rare and serious complication of Actinomadura pelletieri actinomycetoma. JMM Case Rep 2015;2:x

6 Cherian RS, Betty M, Manipadam MT, et al. The "dot-in-circle" sign-a characteristic MRI finding in mycetoma foot: a report of three cases. Br J Radiol 2009;82(980):662-665

7 Reis CMS, Reis-Filho EGM. Mycetomas: an epidemiological, etiological, clinical, laboratory and therapeutic review. An Bras Dermatol 2018;93(1):8-18

8 Fahal AH, Suliman SH, Hay R. Mycetoma: the spectrum of clinical presentation. Trop Med Infect Dis 2018;3(3):97

9 Sarris I, Berendt AR, Athanasous N, Ostlere SJ; OSIRIS collaborative study group. MRI of mycetoma of the foot: two cases demonstrating the dot-in-circle sign. Skeletal Radiol 2003;32(3):179-183
10 Fahal AH, Sheik HE, Homeida MM, Arabi YE, Mahgoub ES. Ultrasonographic imaging of mycetoma. Br J Surg 1997;84(8):1120-1122

11 Laohawiriyakamol T, Tanutit P, Kanjanapradit K, Hongsakul K, Ehara S. The "dot-in-circle" sign in musculoskeletal mycetoma on magnetic resonance imaging and ultrasonography. Springerplus 2014;3:671

12 Abd El-Bagi ME, Fahal AH. Mycetoma revisited. Incidence of various radiographic signs. Saudi Med J 2009;30(4):529-533

13 Neyaz Z, Mohindra N, Bhatnagar A, Marak RSK. Case 249: intramuscular mycetoma. Radiology 2018;286(1):353-359

14 Sen A, Pillay RS. Case report: dot-in-circle sign-an MRI and USG sign for "Madura foot" Indian J Radiol Imaging 2011;21(4):264-266

15 Gupta S, Jain K, Parmar C, Shah P, Raval RC. Mycetoma: nonvenereal perineal lesions. Indian J Sex Transm Dis AIDS 2010;31(1):39-41

16 Relhan V, Mahajan K, Agarwal P, Garg VK. Mycetoma: an update. Indian J Dermatol 2017;62(4):332-340 